\title{
Isotopes of carbon, water and geotracers in paleoclimate research
}

\author{
Fortunat Joos', B. Otto-Bliesner² and E. Brady ${ }^{2}$
}

Bern, Switzerland, 26-28 August 2013

\begin{abstract}
Isotopes of water, carbon, oxygen, and of rare earth elements offer the opportunity to quantitatively understand physical and biogeochemical processes in the Earth System and to unravel past and modern climate change. Past climate and greenhouse gas variations provide insight into how climate might evolve under ongoing anthropogenic forcing. Yet, our understanding of past climate and biogeochemical variations over glacial-interglacial time scales, of past abrupt climate swings, or of climate variability during the last few millennia is still limited. The mechanisms of past climate change are intensively debated. What drives glacial inceptions and terminations? How and why did precipitation vary in the monsoon system and at high latitudes? How did ocean circulation evolve? What regulated the reconstructed variations in atmospheric $\mathrm{CO}_{2}$ and other greenhouse gases?
\end{abstract}

The obstacles to exploiting the full potential of isotopes in order to answer these questions include the scarcity of measurements and of easily accessible and quality-flagged isotope data compilations from paleoclimate archives, incomplete understanding and corresponding uncertainties in isotope process parameterizations in Earth System models, and a lack of systematic multi-model model-data intercomparisons for isotopes.

More than 80 participants attended this conference organized by the Oeschger Centre for Climate Change Research and the Climate and Environmental Physics group at the University of Bern in collaboration with the CESM
Paleoclimate Working Group at the National Center for Atmospheric Research. The meeting is intended to be the fist in a series of conferences. The objective is to provide a networking platform for specialists from the data and modeling communities to integrate information from isotopes across the Earth System components and scientific disciplines, to assess the state-of-the art, and to identify and address knowledge gaps.

The presentations as well as the many outstanding posters addressed a broad set of topics including using water isotopes in speleothems to reconstruct temperature and precipitation changes; using carbon and water isotopes in ice cores and marine sediments to understand past climatic and biogeochemical changes (e.g. Fig. 1); the constraints on past ocean circulation from rare earth and carbon isotopes; $\mathrm{N}_{2} \mathrm{O}$ and nitrogen isotopes in ice cores; how carbon and water isotopes in tree rings can be used to identify physiological mechanisms and document environmental change; the representation of isotopes in models; the interpretation of reconstructed changes with the help of models; the exploitation of synergies between process models, contemporary observations, Earth System Models and paleodata; and the use of present-day monitoring and dedicated process studies for the interpretation of proxy data.

As highlighted in the presentations, much progress has been made in recent years to reconstruct isotopic composition in various archives. Improved proxy records are emerging that extend the previous scope in spatial and temporal resolution and will permit us to draw a more and more detailed picture on their spatio-temporal evolution. At the same time, isotopes of carbon and water and of rare earth elements are increasingly implemented as a standard module in comprehensive Earth System Models and in Earth System Models of Intermediate Complexity.

Concluding discussions revolved around the best way to organize and quality flag existing isotope data to make them more easily accessible to the modeling and experimental communities. It is expected that the meeting stimulated bottom-up research initiatives among the participants that will foster progress on the use of various geotracers in paleoclimate research, in particular in the form of new collaborative efforts that combine information from models and proxies. A follow-up conference is planned to be held in Paris, following the template of this first event in Bern.

\section{ACKNOWLEDGEMENTS}

The organizers thank the Oeschger Centre for Climate Change Research, PAGES, and the Johanna Dürmüller-Bol Foundation for the generous financial support that made this workshop possible. More information, including the abstracts and program, is available at www.oeschger. unibe.ch/events/conferences/isotopes/

\section{AFFILIATIONS}

'Oeschger Centre for Climate Change Research and Physics Institute, University of Bern, Switzerland ${ }^{2}$ National Center for Atmospheric Research (NCAR), Boulder, USA

CONTACT

Fortunat Joos: joos@climate.unibe.ch
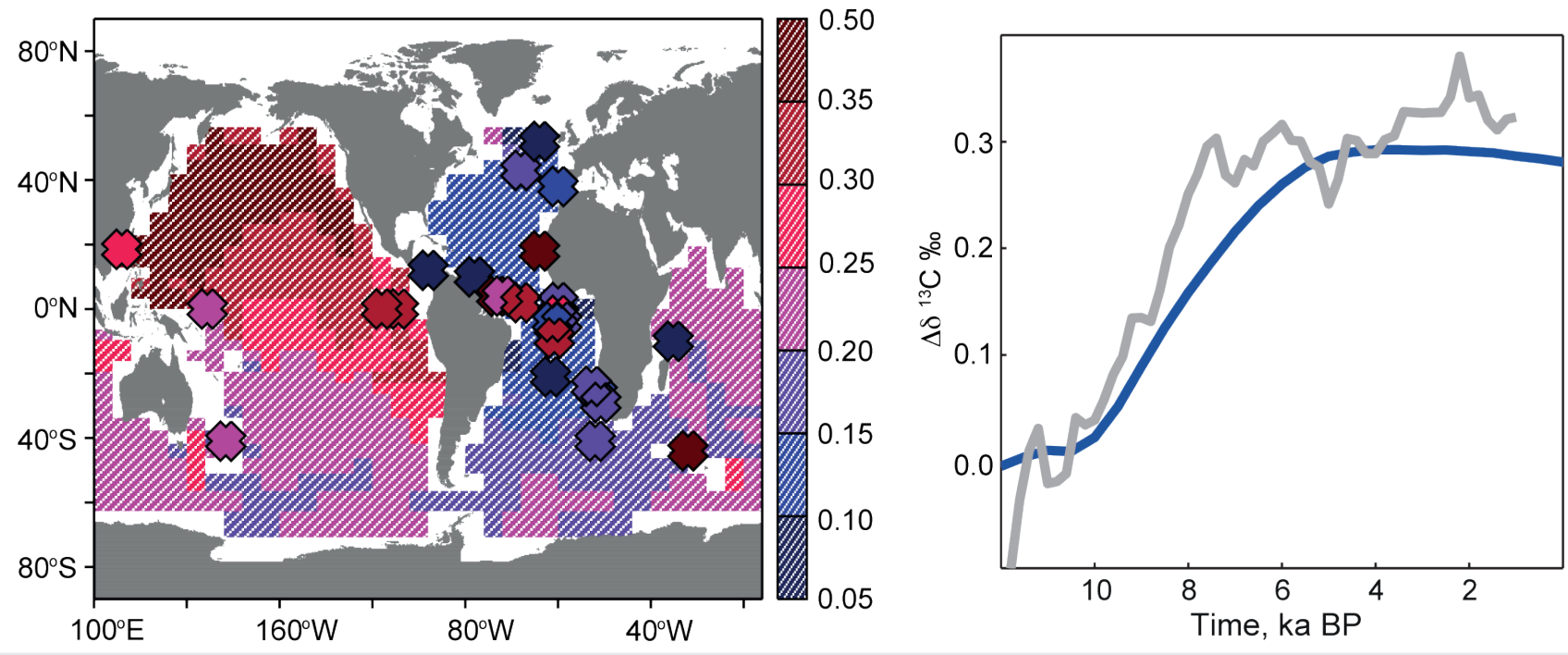

Figure 1: Spatio-temporal evolution of $\delta^{13} \mathrm{C}$ in the deep ocean $(>3000 \mathrm{~m}$ ) as reconstructed from ocean sediment data (symbols) and simulated with an Earth System Model of Intermediate Complexity. The map shows the total change over the Holocene; the right panel shows the evolution from the stacked proxy data (grey) and the globallyaveraged model results (blue). 